\title{
The Feasibility of Using Laser Doppler Vibrometer Measurements from a Passing Vehicle for Bridge Damage Detection
}

\author{
Abdollah Malekjafarian (D), Daniel Martinez $(\mathbb{D}$, and Eugene J. OBrien \\ School of Civil Engineering, University College Dublin, Dublin 4, Ireland \\ Correspondence should be addressed to Daniel Martinez; daniel.martinezotero@ucd.ie
}

Received 24 January 2018; Accepted 8 April 2018; Published 16 May 2018

Academic Editor: Luca Z. Fragonara

Copyright (C) 2018 Abdollah Malekjafarian et al. This is an open access article distributed under the Creative Commons Attribution License, which permits unrestricted use, distribution, and reproduction in any medium, provided the original work is properly cited.

\begin{abstract}
This paper investigates the feasibility of detecting local damage in a bridge using Laser Doppler Vibrometer (LDV) measurements taken from a vehicle as it passes over the bridge. Six LDVs are simulated numerically on a moving vehicle, collecting relative velocity data between the vehicle and the bridge. It is shown that Instantaneous Curvature (IC) at a moving reference, which is the curvature of the bridge at an instant in time, is sensitive to local damage. The vehicle measures Rate of Instantaneous Curvature (RIC), defined as the first derivative of IC with respect to time. A moving average filter is found to reduce the effects of noise on the RIC data. A comparison of filtered RIC measurements in healthy and damaged bridges shows that local damage can be detected well with noise-free measurements and can still be detected in the presence of noise.
\end{abstract}

\section{Introduction}

Highway bridges are important components of transport infrastructure. Aging, environmental conditions, and increased loading can cause deterioration in their lifetime but damage from bridge strikes due to vehicles passing underneath is of particular concern [1]. In recent years, many methods have been used by road owners to provide an acceptable level of safety [2-5]. Visual inspection is the most frequently used approach to bridge damage detection. However, this method can be expensive with the result that it may not be done with sufficient frequency [6]. Several examples exist of direct instrumentation of bridges for structural health monitoring (SHM) purposes [7-10]. Many sensors (e.g., accelerometers or strain gauges) are installed directly on the bridge and the bridge condition is evaluated using the response measured at these sensors. Despite its reliability and efficacy, direct instrumentation has several drawbacks. The most notable is that many systems of sensors, with associated data acquisition systems, are needed to monitor a large network of bridges.
The idea of indirect (or drive-by) bridge health monitoring is first proposed by Yang et al. [11, 12]. In this method, the bridge condition is assessed using the response measured on a passing vehicle $[13,14]$. Several studies have used indirect measurements to estimate the bridge modal parameters such as natural frequencies $[15,16]$ and mode shapes [17, 18]. Malekjafarian et al. [13] provide a critical review of indirect methods published up to 2015. Yang and Chen [19] propose a novel method for estimating the bridge natural frequencies from the responses measured on a vehicle based on the Stochastic Subspace Identification (SSI) method which is claimed to be more effective than conventional methods. Nagayama et al. [20] use a crossspectrum of the signals measured from different vehicles. A higher peak corresponding to the bridge natural frequency is obtained compared to the power spectral density (PSD). González et al. [21] use a Half-Car model over a finite element bridge to obtain the damping value on a simply supported beam. Damping is identified as the value that minimizes the error function. Malekjafarian and OBrien [22] use drive-by measurements to estimate the bridge mode 
shapes. OBrien and Malekjafarian [17] introduce a mode shape-based damage detection algorithm using the response measured by laser vibrometers installed on a passing vehicle. Kong et al. [23] present a tractor towing two trailers crossing a bridge. They show an optimized vehicle configuration for drive-by estimation of bridge natural frequencies and mode shapes.

OBrien et al. [24] propose a drive-by damage detection method using empirical mode decomposition (EMD) of the accelerations measured on a passing vehicle. It is shown that some components of the signal include damage information. Hester and González [25] employ a wavelet transform of the signal measured on a vehicle for the localization of bridge damage. McGetrick et al. [26] use Global Navigation Satellite Systems (GNSS) in a drive-by monitoring approach. The potential of smartphones to measure accelerations is investigated by comparing the results to the accelerations obtained through sensors installed in the vehicle. Kim et al. [27] develop a laboratory test consisting of a trucktrailer system. They show that the effect of road profile can be reduced by subtracting the signals measured from two following axles. Elhattab et al. [28] obtain bridge displacement profile differences using accelerations measured from a passing vehicle for damage detection. The approach is able to detect localized damage, but vehicle calibration is needed to use the method in field applications. OBrien et al. [29] propose using curvatures of the bridge deflections at a moving coordinate for damage detection. They assume that the bridge deflection can be measured from a passing vehicle. OBrien et al. [30] propose the use of a Traffic Speed Deflectometer (TSD) for drive-by bridge damage detection. A TSD [31] is a specialized vehicle which is used to measure the deflection "basin" (depression) in a road pavement due to a heavy axle load. It is equipped with several laser vibrometers which measure relative velocities between the road pavement and the vehicle [32].

In this paper, a local damage detection method is proposed using relative velocities such as those measured on a TSD. The method is based on the concept of Instantaneous Curvature (IC). It is shown that curvature is sensitive to damage and can detect the presence and location of damage $[14,29,30]$. Since velocities are measured on a TSD, Rate of Instantaneous Curvature (RIC), the first time derivative of IC, is introduced for damage detection. Data such as that which would be collected from Laser Doppler Vibrometers (LDVs) are used for RIC calculation. A numerical case study of a TSD passing over a simply supported bridge with a class A road roughness is used. Two damage scenarios including single damage and multiple damage points are considered. Two damage indicators are proposed using the concept of RIC. The effectiveness of the proposed indicators is evaluated for noisy measurements.

\section{Numerical Modelling}

2.1. Vehicle Bridge Interaction Model. A numerical model of vehicle bridge interaction is implemented using the finite element (FE) method (Figure 1) in MATLAB. A Half-Car model is used here to represent the TSD. The model has
Table 1: Properties of the vehicle.

\begin{tabular}{lcc}
\hline Half-Car property & Notation & Value \\
\hline Weight of the sprung mass & $m_{s}$ & $16.2 \mathrm{t}$ \\
Unsprung mass axle 1 & $m_{u 1}$ & $900 \mathrm{~kg}$ \\
Unsprung mass axle 2 & $m_{u 2}$ & $900 \mathrm{~kg}$ \\
Length of the vehicle & $L_{v}$ & $11.25 \mathrm{~m}$ \\
Tyre 1 stiffness & $K_{t, 1}$ & $1.75 \times 10^{6} \mathrm{~N} / \mathrm{m}$ \\
Tyre 2 stiffness & $K_{t, 2}$ & $3.5 \times 10^{6} \mathrm{~N} / \mathrm{m}$ \\
Damper 1 stiffness & $K_{s, 1}$ & $4 \times 10^{5} \mathrm{~N} / \mathrm{m}$ \\
Damper 2 stiffness & $K_{s, 2}$ & $10^{6} \mathrm{~N} / \mathrm{m}$ \\
Damper 1 damping & $C_{s, 1}$ & $10^{4} \mathrm{Ns} / \mathrm{m}$ \\
Damper 2 damping & $C_{s, 2}$ & $2 \times 10^{4} \mathrm{Ns} / \mathrm{m}$ \\
Body mass frequency & $f_{\text {body }}$ & $1.27 \mathrm{~Hz}$ \\
Axle 1 mass frequency & $f_{\text {axle1 }}$ & $7.80 \mathrm{~Hz}$ \\
Axle 2 mass frequency & $f_{\text {axle2 }}$ & $11.28 \mathrm{~Hz}$ \\
Centre of gravity distance from axle 1 & $D_{1}$ & $4.71 \mathrm{~m}$ \\
Centre of gravity distance from axle 2 & $D_{2}$ & $2.89 \mathrm{~m}$ \\
\hline
\end{tabular}

TABle 2: Properties of the bridge.

\begin{tabular}{lcc}
\hline Bridge property & Notation & Value \\
\hline Number of elements & $N$ & 200 \\
Length & $L$ & $20 \mathrm{~m}$ \\
Young's modulus & $E$ & $3.5 \times 10^{10} \mathrm{~N} / \mathrm{m}^{2}$ \\
2nd moment of area & $I$ & $1.26 \mathrm{~m}^{4}$ \\
Bridge depth & $h$ & $1 \mathrm{~m}$ \\
Mass per unit length & $\mu$ & $37,500 \mathrm{~kg} / \mathrm{m}$ \\
Damping & $\xi$ & $3 \%$ \\
First natural frequency & $f_{1}$ & $4.26 \mathrm{~Hz}$ \\
Length of the approach & $L_{\text {app }}$ & $10 \mathrm{~m}$ \\
\hline
\end{tabular}

4 independent degrees of freedom (DOFs) corresponding to body mass vertical translation $\left(v_{s}\right)$, body pitch rotation $\left(\theta_{s}\right)$, and two vertical axle translations $\left(v_{u, 1}\right.$ and $\left.v_{u, 2}\right)$. The vehicle body mass is $m_{s}$ and $I_{s}$ is its moment of inertia. Two unsprung masses, $m_{u, 1}$ and $m_{u, 2}$, between the tyres and the suspension, represent the axle weights. These unsprung masses are connected to the road by linear springs simulating tyre stiffnesses, $K_{t, 1}$ and $K_{t, 2}$. The sprung and unsprung masses are connected by a combination of linear springs, $K_{s, 1}$ and $K_{s, 2}$, and viscous dampers, $C_{s, 1}$ and $C_{s, 2}$. The properties of the vehicle are given in Table 1.

The bridge is modelled as a simply supported beam using 200 discretized elements. Each element has 4 DOFs (one translation and one rotation per node). The properties of the beam are listed in Table 2. Viscous damping is generally accepted to model the complex behaviour of a bridge [33-35]. Rayleigh damping is adopted here to model viscous damping of the bridge using its mass and stiffness matrices with

$$
C_{b}=\alpha M_{b}+\beta K_{b}
$$




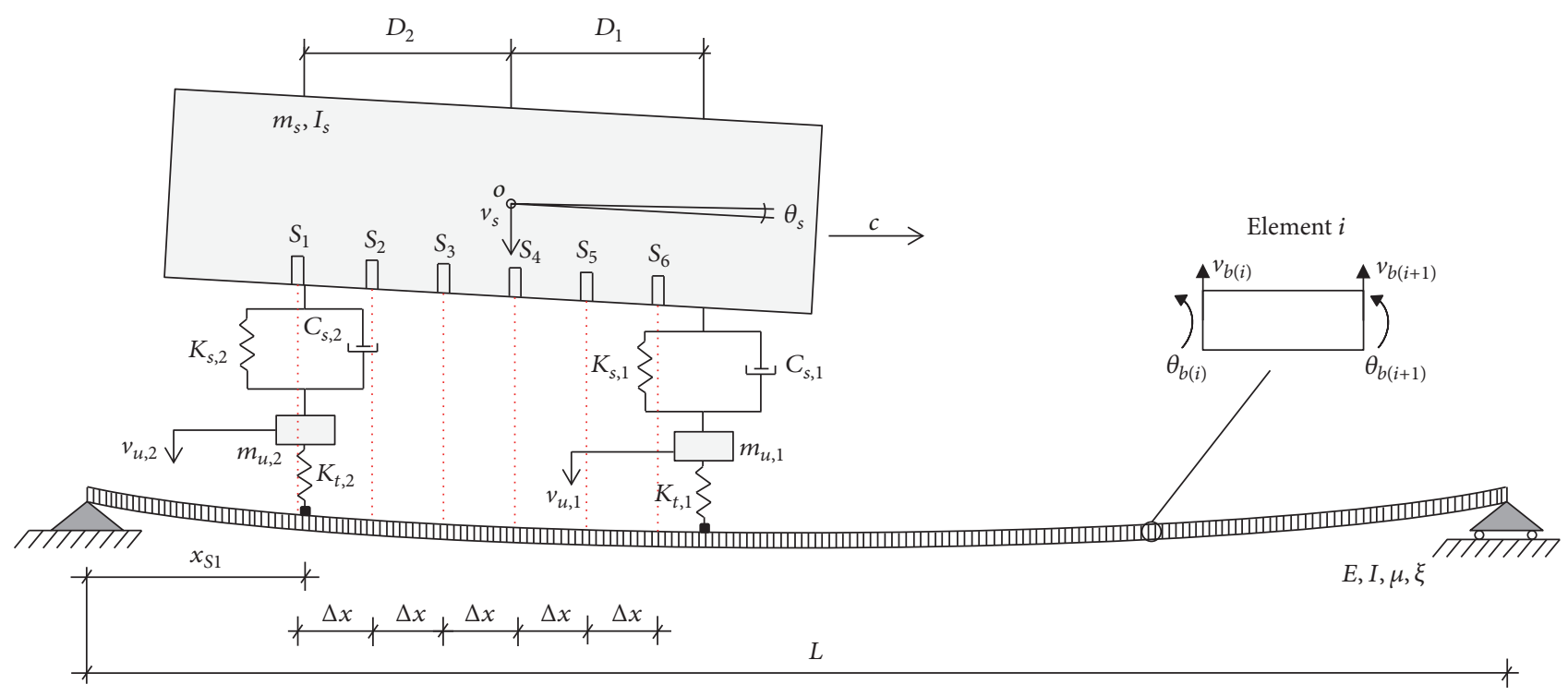

FIGURE 1: Vehicle bridge interaction model ( $S_{1}$ to $S_{6}$ represent the laser vibrometers).

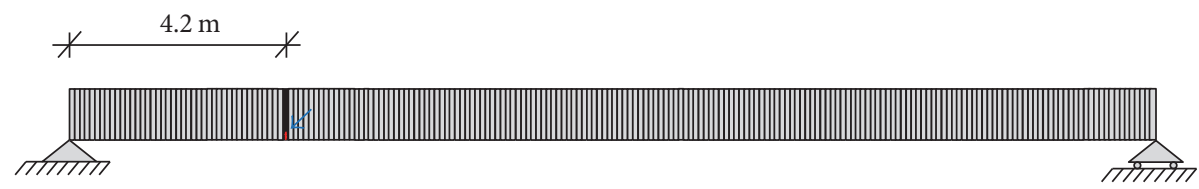

(a)

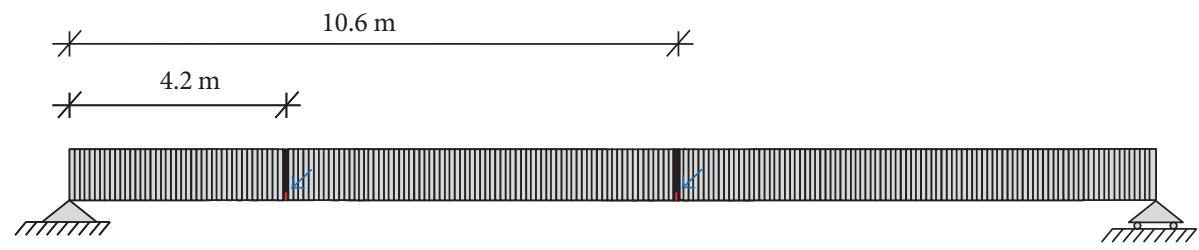

(b)

Figure 2: Damage scenarios: (a) single damage case and (b) multiple damage case.

where $\alpha$ and $\beta$ are constants estimated from the two main natural frequencies, $\omega_{1}$ and $\omega_{2}$, of the bridge and the damping ratio, $\xi[36]$ :

$$
\begin{aligned}
& \alpha=\frac{2 \xi \omega_{1} \omega_{2}}{\omega_{1}+\omega_{2}} \\
& \beta=\frac{2 \xi}{\omega_{1}+\omega_{2}} .
\end{aligned}
$$

A bridge damping ratio of $3 \%$ is assumed in this study.

The vehicle and bridge are coupled at the tyre contact point. The equation of motion of the coupled system is

$$
M_{g} \ddot{v}+C_{g} \dot{v}+K_{g} v=F,
$$

where $M_{g}, C_{g}$, and $K_{g}$ represent the global matrix mass, damping and stiffness matrices and $F$ represents the force vector. The equation of motion of the coupled system is solved using the Wilson-Theta integration method at each time step [37]. Unconditional stability of the integration is achieved by setting $\theta=1.420815$. The model is implemented using a sampling frequency of $1000 \mathrm{~Hz}$.

Six Laser Doppler Vibrometers (LDVs) are taken to be installed on the TSD, as illustrated in Figure 1. Such sensors measure the relative velocity between the vehicle and the bridge, $\dot{q}=\dot{v}-\dot{v}_{\text {veh }}$.

2.2. Damage Modelling. The damage in the bridge is modelled using the method described by Sinha [38]. Distress in the locations close to the crack is considered, resulting in a progressive loss of stiffness. The model assumes a linear decrease in stiffness over a distance of 3 times the bridge depth, $h$, resulting in a triangular reduction that is zero at a distance of $1.5 h$ on either side of the crack and maximum at the crack location.

Two damage scenarios are used in this research. The first one has a single crack at $4.2 \mathrm{~m}$ from the left support (Figure 2(a)) and the second has two cracks, at $4.2 \mathrm{~m}$ and $10.6 \mathrm{~m}$ (Figure 2(b)). A crack depth equal to $20 \%$ of the 


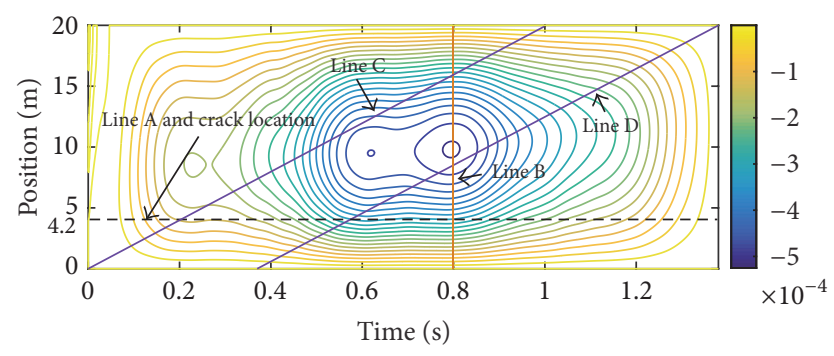

FIGURE 3: Contour plot of deflections (in $\mathrm{m}$ ) for the damaged bridge for single damage scenario in Figure 2(a).

total depth of the bridge is considered at each location. This equates to a maximum loss of stiffness of approximately $50 \%$ at the damage location.

\section{Instantaneous Curvature}

Curvature is commonly approximated as the ratio of bending moment to stiffness [39]. Consequently, a local increase in curvature is expected at a damage location due to the local loss of stiffness. In this section, bridge deflection is considered versus time and position, as the vehicle is passing over it.

3.1. Bridge Deflection versus Time and Position. The TSD is simulated to pass over the healthy and damaged bridges at a speed of $c=20 \mathrm{~m} / \mathrm{s}$. The first damage scenario shown in Figure 2(a) is considered. The deflections are calculated at all positions on the bridge at each time step. A contour plot of the damaged bridge deflection is shown in Figure 3. No road profile is considered in this example.

Bridge deflections can be measured at a fixed point (e.g., using laser-based systems). He and Zhu [40] show that the bridge deflection response at a fixed point to a moving load can be used for damage detection. Such a measurement can be represented by line A in Figure 3. Figure 4(a) shows the deflections at a fixed point on the beam for the healthy and damaged cases. There are peaks, partly due to vibration and partly due to the axles passing the "sensor" location. The bridge deflections can also be represented at a point in time. Line B in Figure 3 represents this measurement. Figure 4(b) shows the deflection at a fixed point in time (corresponding to line B) as would be captured by a camera measurement system. In this way, the vehicle is located at the same location in the damaged and healthy cases and there is a difference in the deflections due to damage. Such a measurement would need something like a high-quality camera that can cover the whole bridge in one shot which is infeasible at the required level of accuracy at this time.

OBrien et al. [24] show that the bridge deflection response to a passing vehicle at a moving coordinate provides the damage location. This measurement type is represented by lines $\mathrm{C}$ and $\mathrm{D}$ in Figure 3. Figure 5 shows the bridge deflection signal for a moving reference-line $\mathrm{D}$ - as would be recorded by a vehicle travelling at constant speed measuring relative to a perfectly horizontal reference line. There is a clear difference between the healthy and damaged bridge models and the difference is greatest in the region of the damage location. The challenge with this method is that it is not possible to measure the bridge absolute deflection directly from the passing vehicle.

3.2. Curvature. Curvature at each point in time can be estimated from deflection measurements at three different positions on the bridge [29]. Figure 6 shows a contour plot of deflections for the healthy bridge without considering a road profile. A system of 3 equally spaced sensors (3 yellow points) passing at constant speed over the healthy bridge is illustrated. Instantaneous Curvature (IC) is the second derivative of deflection at a given instant and is estimated here as the difference of the differences. In this case, it could be calculated using the deflections from the 3 yellow points shown.

Figure 7 shows the IC for the healthy and damaged cases when the measurements are centered on the second axle of the vehicle. The $x$-axis in the figure shows the position of the middle yellow point (middle sensor). A sharp difference near the crack location is clear, as expected due to the local loss of stiffness. By comparing Figures 5 and 7, it can be concluded that IC provides more local information about damage.

\section{Damage Detection Using Velocity Measurements}

Although IC is a good damage indicator, it is infeasible at this time to measure bridge deflection accurately at a moving reference. However, the TSD is equipped with several laser vibrometers which can measure the relative velocity between vehicle and bridge at several points (see Figure 1). Such a relative velocity contains the vehicle and bridge response components. Rate of Instantaneous Curvature (RIC) is introduced as a new parameter in this section based on the concept of IC. Two damage indicators are proposed based on RIC.

To estimate the Rate of Instantaneous Curvature, three laser vibrometers are required:

$$
\operatorname{RIC}(x, t)=\frac{\dot{q}(x-\Delta x, t)-2 \dot{q}(x, t)+\dot{q}(x+\Delta x, t)}{\Delta x^{2}},
$$

where $\dot{q}(x, t)$ is the relative velocity (between vehicle and bridge) when the central laser vibrometer is located at a distance of $x$ from the left end of the bridge. As there are six sensors available, four combinations of the sensors can be taken for RIC (sensors 1, 2, and 3, sensors 2, 3, 4, sensors 3, 4, and 5 , and sensors 4, 5, and 6). To increase the accuracy of the measurement, an average of these four measurements, $\mathrm{RIC}_{\mathrm{av}}$, is used.

Two variations of the damage indicator are proposed based on $\mathrm{RIC}_{\mathrm{av}}$. Difference Ratio (DR) is based on the difference between the $\mathrm{RIC}_{\mathrm{av}}$ values for the healthy and damaged bridges at each point:

$$
\operatorname{DR}(x)=\frac{\operatorname{RIC}_{\mathrm{av}}^{\mathrm{dam}}(x)-\mathrm{RIC}_{\mathrm{av}}^{\text {hea }}(x)}{\min \left(\mathrm{RIC}_{\mathrm{av}}^{\text {hea }}\right)} \times 100(\%),
$$




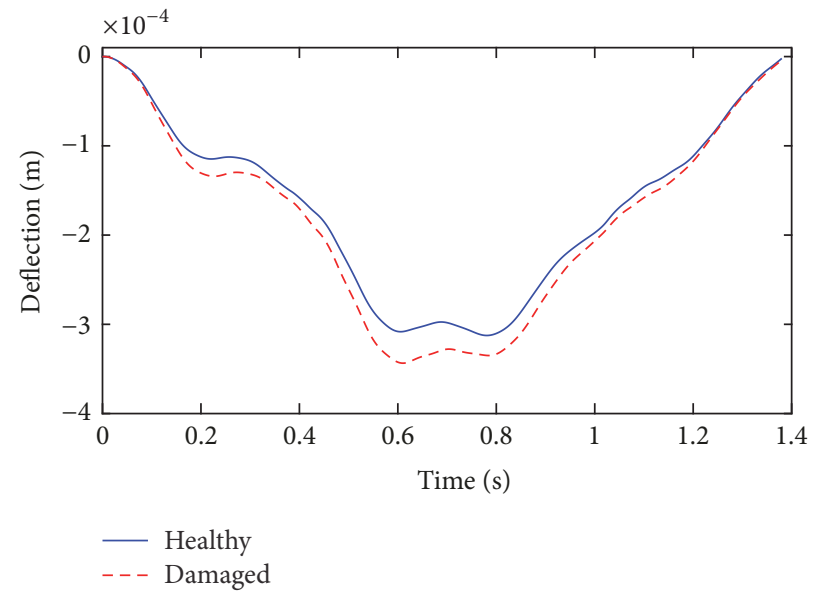

(a)

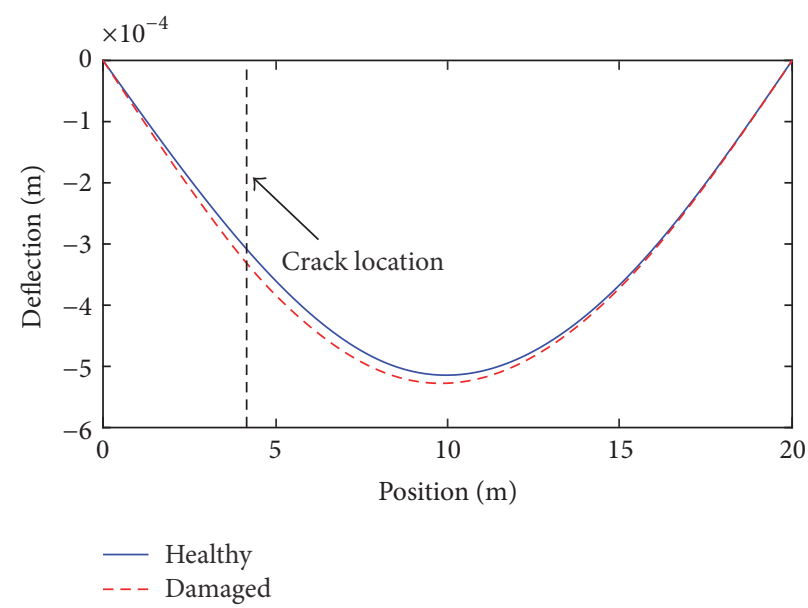

(b)

Figure 4: Cross sections through the contour plot of deflections in Figure 2(a): (a) line A, constant "sensor" position at $4.2 \mathrm{~m}$; (b) line B, constant time at $0.8 \mathrm{~s}$.

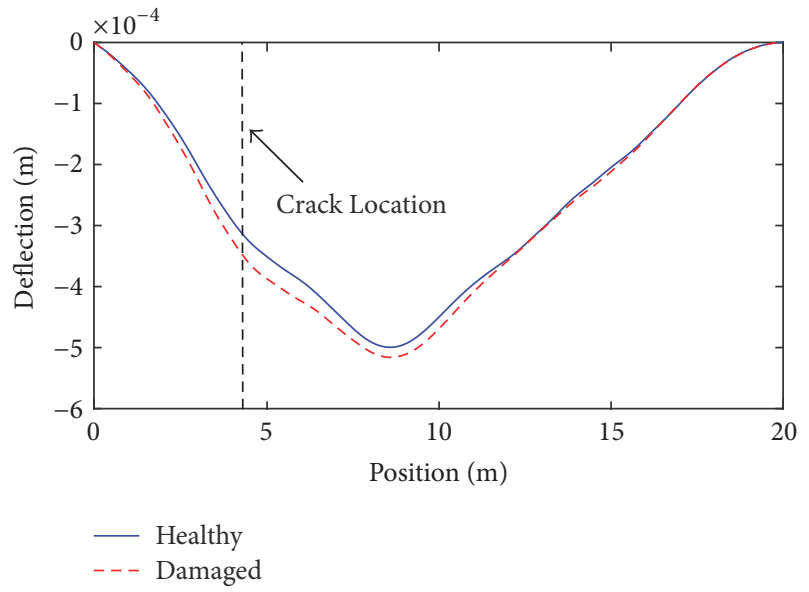

Figure 5: Cross section of the contour plot at line D (moving reference).

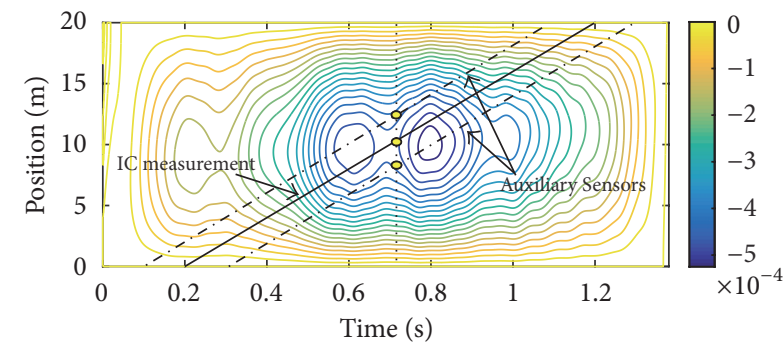

FIGURE 6: Instantaneous Curvature calculation using three deflection measurements on the healthy bridge.

where $\mathrm{RIC}_{\mathrm{av}}^{\text {hea }}$ and $\mathrm{RIC}_{\mathrm{av}}^{\mathrm{dam}}$ are the average $\mathrm{RIC}$ values for the healthy and damaged bridges, respectively. In the second variation, a moving average filter is applied to these functions to remove high frequency effects:

$$
\operatorname{MAD}(x)=\frac{(1 / z) \sum_{i=-(z-1) / 2}^{i=(z-1) / 2} \operatorname{RIC}_{\mathrm{av}}^{\mathrm{dam}}(x+i d x)-(1 / z) \sum_{i=-(z-1) / 2}^{i=(z-1) / 2} \mathrm{RIC}_{\mathrm{av}}^{\text {hea }}(x+i d x)}{\min \left(\mathrm{RIC}_{\mathrm{av}}^{\mathrm{hea}}\right)} \times 100(\%)
$$

where $z$ is the number of points used for the moving average and $d x$ is the sampling interval in space, defined by $d x=c / f_{s}$, where $c$ is the vehicle's speed and $f_{s}$ is the sampling frequency. A value of $z=51$ is considered in this study which averages over $1 \mathrm{~m}$ (equal to the bridge depth in this example) around each point.

\section{Numerical Case Study}

5.1. Noise-Free Measurements. The numerical model outlined in Section 2 is employed here. A class " $A$ " road profile is introduced, as is typical of a highway in good condition [41].
It is generally assumed that profiles in adjacent tracks of a vehicle in a lane are well correlated, reducing the change in dynamic excitation. Other changes in the road profile caused by environmental issues such as spring thaw in cold climates are much less likely in a bridge than on a regular road foundation. However, there is a risk that local profile damage, such as a pothole, may be confused as bridge damage.

The same two damage scenarios as used in Section 2 are considered. Relative velocities are noted from six laser vibrometers on the simulated TSD. Four RICs are calculated using (4) and $\mathrm{RIC}_{\mathrm{av}}$ is calculated for the healthy and damaged bridges. Other external factors such as temperature change 


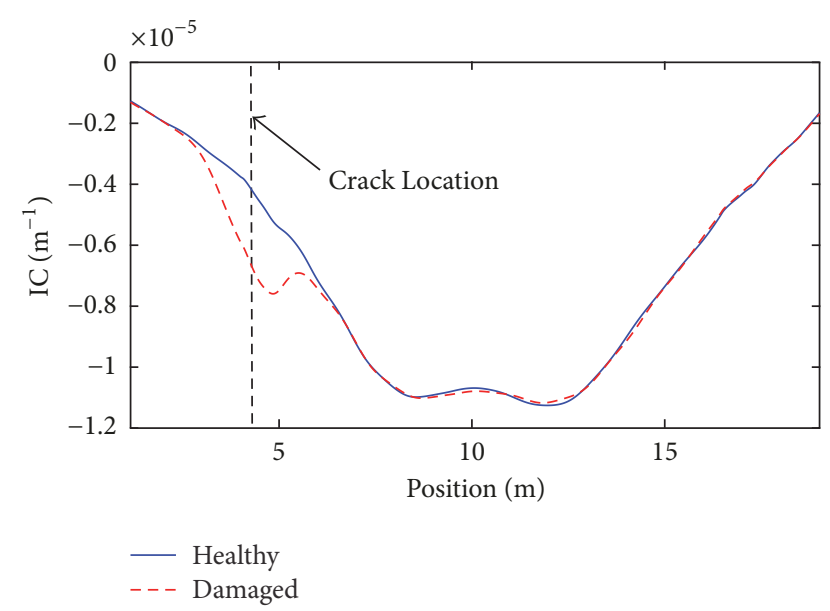

FIgURE 7: IC at the moving reference.

are not considered in the simulation. Axial temperature changes in concrete affect stiffness but in a uniform way and no local drop in stiffness are expected at any particular bridge location. Differential temperature changes (e.g., top heating up relative to bottom of bridge deck) do cause curvature along bridges but do not cause local changes in curvature.

Figures 8(a) and 8(b) show $\mathrm{RIC}_{\mathrm{av}}$ for the single and multiple damage scenarios, respectively. The effect of damage at the 1st location is quite clear at that location for both damage scenarios. RIC $_{\mathrm{av}}$ tends to oscillate, likely due to bridge vibration, and the damage causes a phase shift in that oscillation in the 2 nd half of the bridge. Damage at the 2nd location has some effect (Figure $8(\mathrm{~b})$ ) but it is not as obvious as at the 1st location. Further, there is risk that the effect of damage at the 2 nd location could be confused with the phase shift caused by damage at the 1st location.

Figure 9 shows the damage ratio (DR) for both damage scenarios. For the single damage case, there is a clear peak at the damage location and oscillations in the second half of the bridge, corresponding to the phase shift evident in Figure $8(a)$. For the multiple damage scenario (Figure 9(b)) the second damage close to midspan is not clearly distinguishable from the oscillation.

The moving average filter has been found to be effective in filtering out the oscillation due to the phase shift. Figure 10 shows the MAD for the two damage scenarios. It can be seen that there are reasonable peaks at both damage locations.

\subsection{Influence of Changes in Bridge Boundary Condition.} Boundary conditions in a simply supported bridge may change due to failure of a bearing which may result in it becoming resistant to rotation. In this section, a rotational spring is added to the left support of the bridge to investigate the influence of changes in the bridge boundary conditions on the effectiveness of the proposed method. Two different cases are considered, first with $K_{\mathrm{rs}}=10^{8} \mathrm{Nm}$ and a second with $K_{\mathrm{rs}}=3.15 \times 10^{8} \mathrm{Nm}$, where $K_{\mathrm{rs}}$ is the rotational stiffness added to the left support. This additional component of stiffness is added to the beam stiffness matrix using the procedure explained in [42]. Figure 11 presents MAD using the rotational springs (with nonzero stiffness) as the damage. Differences in values close to the left support can be seen due to the hogging moment generated by the presence of the springs. It is noteworthy that changes in MAD due to bridge damage to the underside of a girder are of opposite sign (corresponding to sagging moment).

MAD is also calculated for the combined effect of a rotational spring and regular (loss of stiffness) damage. Figure 12 shows the influence of adding a rotational spring with stiffness $K_{\mathrm{rs}}=3.15 \times 10^{8} \mathrm{Nm}$. In this case, the change in support condition has little effect on the result. Changes in boundary conditions are not considered in the following sections.

5.3. Influence of Measurement Noise. Although the LDV is known as a very accurate sensor, its signals are still polluted by some levels of noise and there are other sources of inaccuracy that may affect the measurement. LDV noise is a function of sampling frequency [43]. In order to evaluate the effectiveness of the proposed damage indicators in a noisy environment, a noise is added to the relative velocity measurements as follows:

$$
\dot{q}_{\mathrm{ns}}(t)=\dot{q}(t)+K \times \sqrt{f_{s}} \times N_{\mathrm{ns}}
$$

where $\dot{q}_{\mathrm{ns}}(t)$ is the noisy relative velocity, $\dot{q}(t)$ is the original signal, $K$ is a constant value defining the energy in the noise, and $N_{\mathrm{ns}}$ is a random vector with zero mean value and unit standard deviation. $K=10^{-6} \mathrm{~m} / \mathrm{s} \sqrt{\mathrm{Hz}}$ is chosen in this study according to information provided by the designer of the TSD [44].

The simulations are repeated and noisy relative velocities from six sensors are obtained. In order to reduce the influence of the noise on the measurements, a low-pass Blackman window filter is applied [45] to the measurements:

$$
\dot{q}_{\mathrm{fil}}(t)=\sum_{i=1}^{I} \omega_{\mathrm{norm}}[i] \times \dot{q}_{\mathrm{ns}}\left(t+\left(i-\frac{I+1}{2}\right) \times \frac{T}{f}\right),
$$

where $\dot{q}_{\text {fil }}(t)$ is the filtered signal, $\omega_{\text {norm }}[i]$ is the normalized vector of the Blackman window and $T$ is the total time of measurement. $\omega_{\text {norm }}[i]$ is defined by

$$
\omega_{\text {norm }}[i]=\frac{\omega[i]}{\sum_{k=1}^{K} \omega[k]},
$$

where $\omega[k]$ is a component of the vector of the Blackman window, defined by

$$
\omega[k]=0.42-0.5 \cos \left(\frac{2 \pi k}{K-1}\right)+0.08 \sin \left(\frac{4 \pi k}{K-1}\right),
$$

where $K$ is the number of components in the vector $\omega$ and $k$ is the position of the window vector. In this study, a Blackman window of $K=11$ points is used to filter the noisy signals. $\mathrm{RIC}_{\mathrm{av}}$ is obtained from the filtered noisy relative velocity measurements. Figure 13 shows the effect of noise on $\mathrm{RIC}_{\mathrm{av}}$. Although more oscillation can be seen in Figure 13 compared to Figure 8, there are still detectable changes in $\mathrm{RIC}_{\mathrm{av}}$ at the damage locations. 


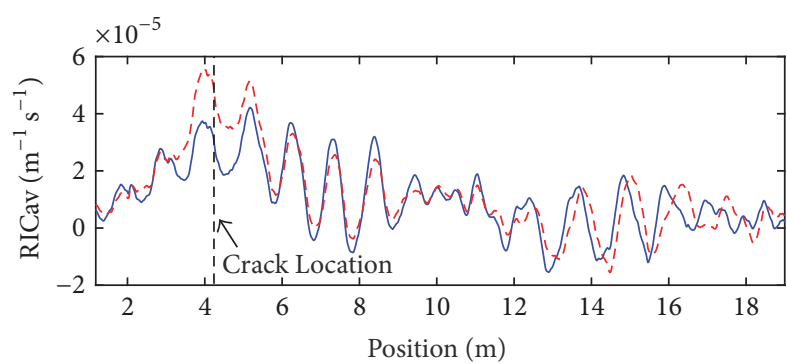

— Healthy

-- - Damaged

(a)

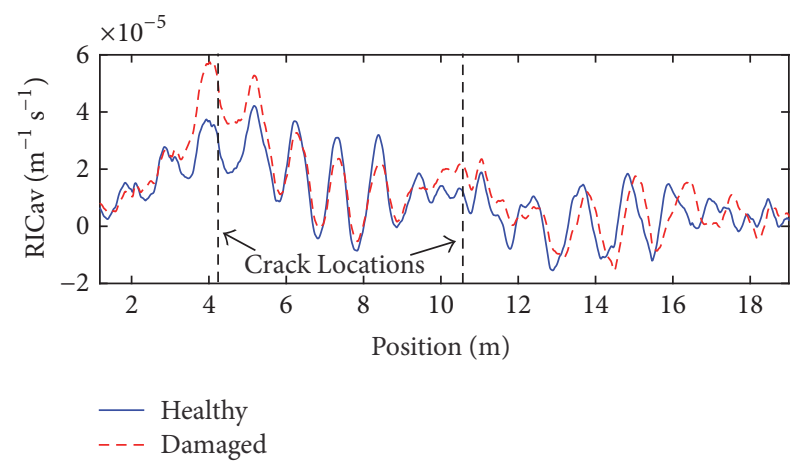

(b)

FIGURE 8: Comparison of $\mathrm{RIC}_{\mathrm{av}}$ for healthy and damaged cases: (a) the first damage case and (b) the second damage case.

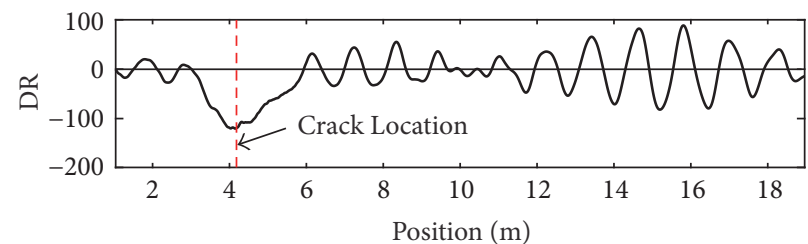

(a)

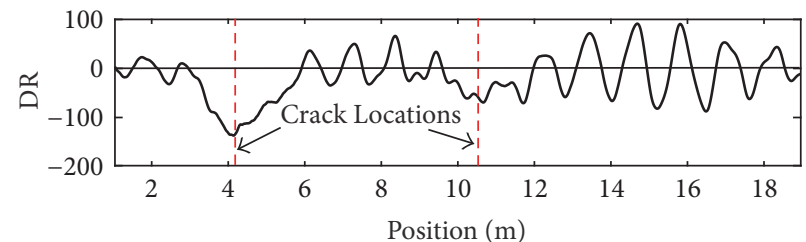

(b)

FIGURE 9: Damage ratio: (a) the first damage case and (b) the second damage case.

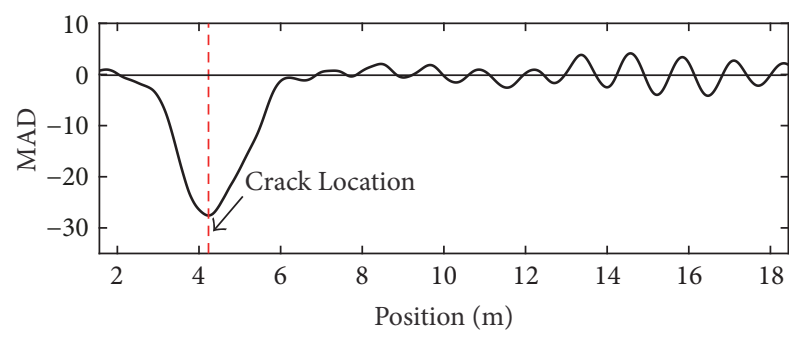

(a)

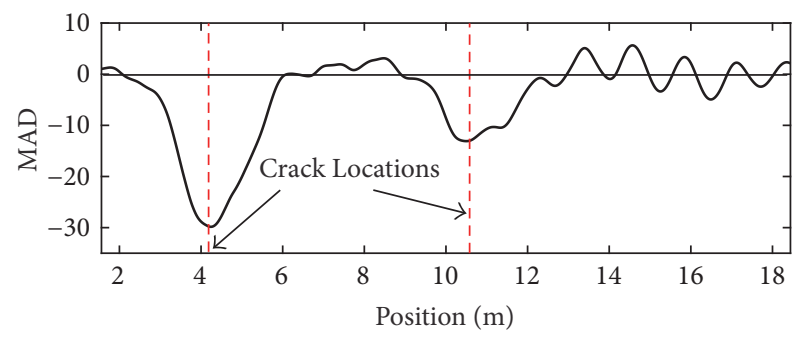

(b)

FIgURE 10: The second damage indicator: (a) the first damage case and (b) the second damage case.

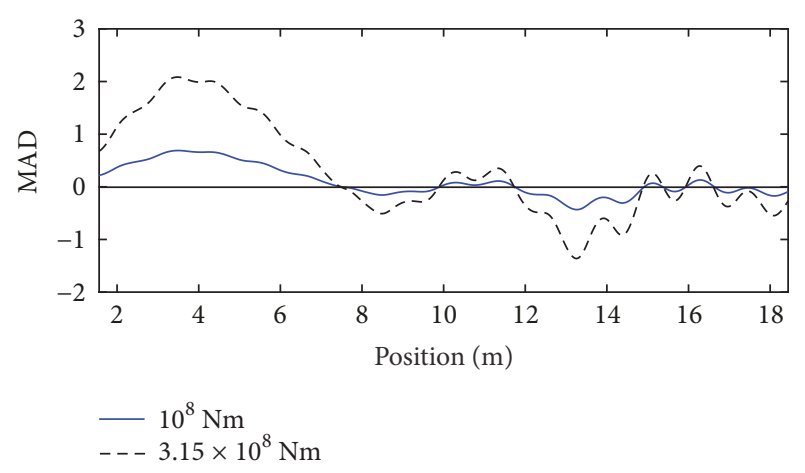

FIGURE 11: Effect of a rotational spring applied to the left support of a simply supported beam.

Figure 14 shows the DR for the noisy measurements. It can be seen that more oscillations are present than in the absence of noise (Figure 9). The location of first damage in both cases is hardly detectable and there is no dominant peak at the location of the second damage in the multiple damage case.

Figure 15 show the results for the filtered results (MAD) in the presence of noise. In this case, the damage locations are detectable in both damage cases with acceptable accuracy. There is a clear peak at the damage location in Figure 15(a). Figure 15(b) shows that even multiple damages can be detected using the MAD. There is a smaller peak at the second damage location compared to the first one.

\section{Discussion}

It is shown here that the proposed damage detection strategy works well in numerical case studies. However, there are some issues that may need to be considered before application in the field. In this paper the influence of additional random 


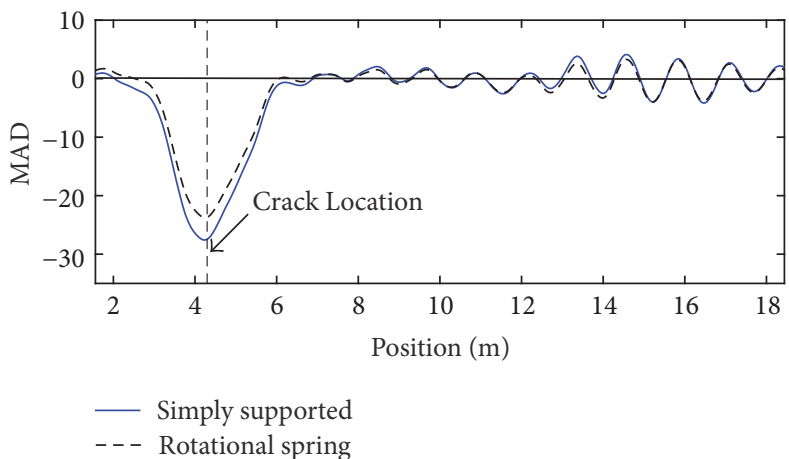

(a)

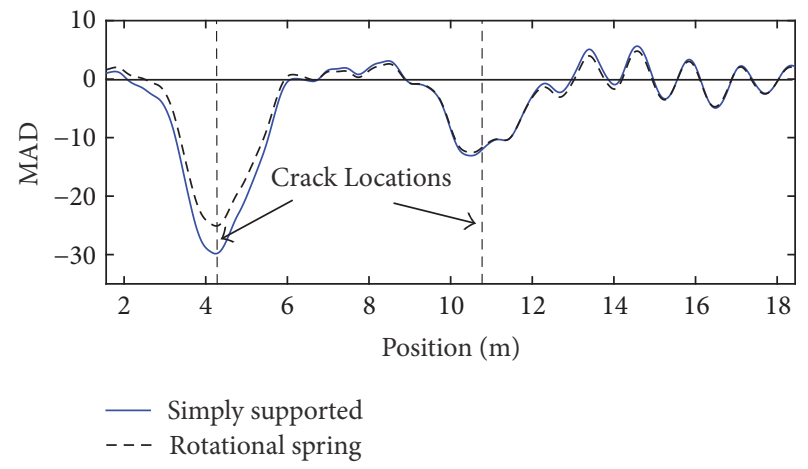

(b)

FIGURE 12: MAD for the bridge with and without a rotational spring: (a) the first damage case and (b) the second damage case.

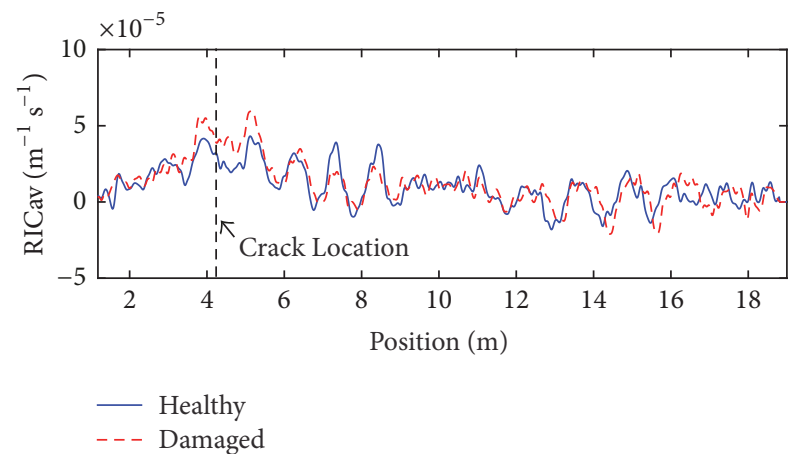

(a)

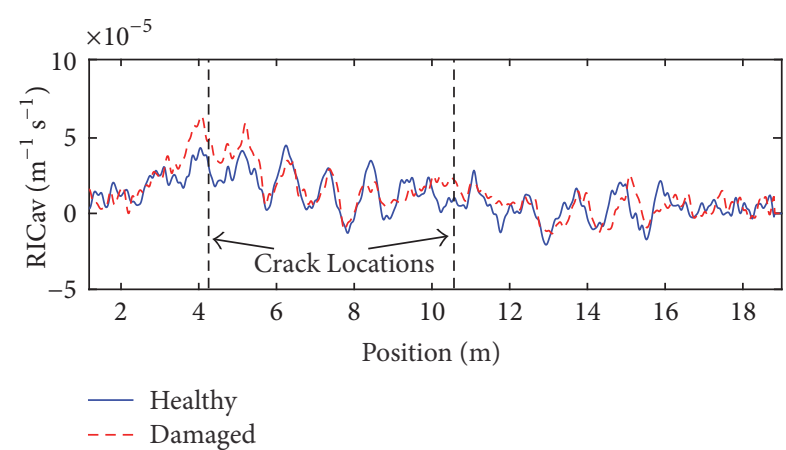

(b)

FIGURE 13: Comparison of RIC ${ }_{\text {av }}$ for healthy and damaged cases with noisy measurements: (a) the first damage case and (b) the second damage case.

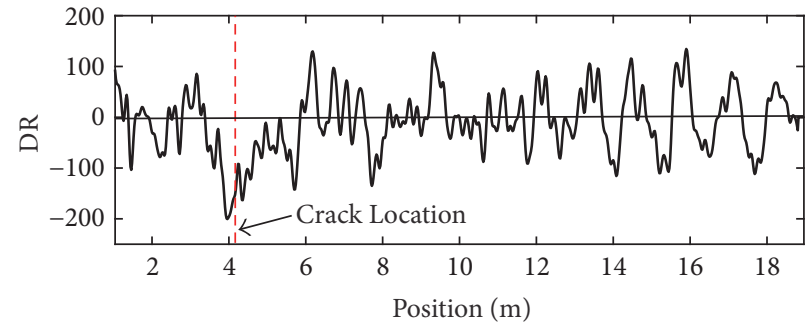

(a)

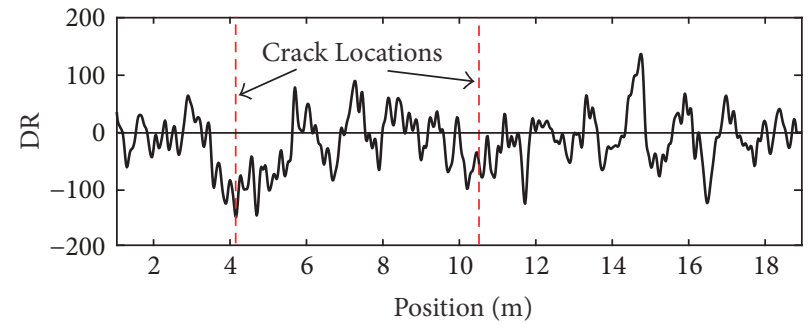

(b)

FIGURE 14: The first damage indicator in presence of noise: (a) the first damage case and (b) the second damage case.

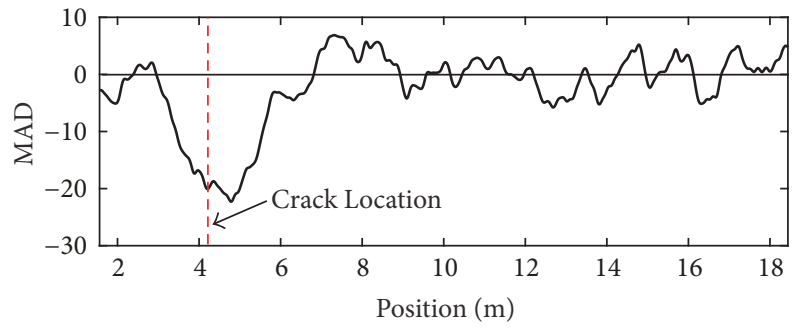

(a)

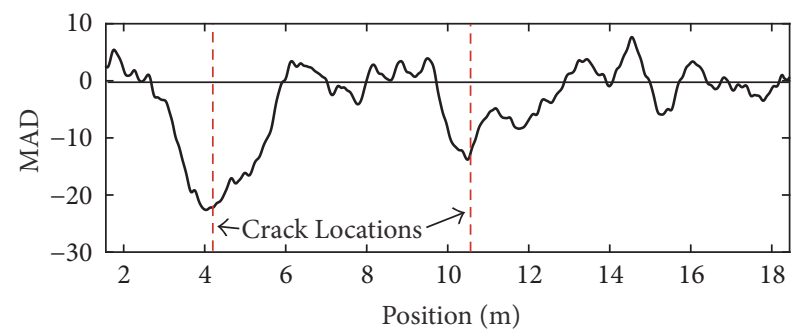

(b)

FIGURE 15: The second damage indicator in presence of noise: (a) the first damage case and (b) the second damage case. 
traffic is not considered. However, it is expected not to affect the ability of RIC to locate damage. Drive-by methods are generally proposed for short and medium span bridges. A short time is spent crossing the bridge, normally between 1 and 2 seconds. Considering that following vehicles are generally more than a second apart, no contamination is expected to affect the measurements from the same-lane vehicle. However, contamination from a vehicle in an adjacent lane in same-direction lanes is likely to be common though the contribution of traffic in one lane to curvature in another lane is relatively small. Furthermore, vehicles in a neighboring lane are usually of lesser weight (e.g., cars) compared to the TSD. Fortunately, no sharp change in curvature is expected to occur due to vehicles in adjacent lanes. While there may be a change in the velocity response, bridge strike damage should still be detectable through the "bump" caused by the local change in curvature.

Wind and boundary motion tend to cause vibration at the bridge natural frequencies. If a frequency is excited that has a similar local effect as damage, this may be a source of increased contamination of the signal. However, wind influence is usually considered for the analysis of long-span bridges (e.g. greater than $100 \mathrm{~m}$ ). As mentioned before, a drive-by strategy usually involves short and medium span bridges. For this reason, wind influence is expected not to create a sharp increase in an unexpected location.

The road profile roughness could be rougher than a class A profile. The expansion joints at the bridge entrance/exit may be damaged and may generate bouncing and/or pitching in the vehicle response. Finally, there may be other, unanticipated sources of inaccuracy which may influence the effectiveness of the proposed method.

\section{Conclusion}

In this paper, a new damage detection approach is proposed using the response measured on a TSD. It is shown that Rate of Instantaneous Curvature (RIC), which is obtained from relative velocities, is sensitive to damage. Two variations of the damage indicator, Difference Ratio (DR) and Moving Average Difference (MAD), are introduced. The effectiveness of the approach in the presence of noise is also investigated. It is shown that MAD provides the damage location in a noisy environment for single and multiple damage scenarios. The numerical results confirm that the proposed approach can be used for drive-by damage detection for a vehicle speed of $20 \mathrm{~m} / \mathrm{s}$.

\section{Data Availability}

This paper can be accessed through UCD repository.

\section{Conflicts of Interest}

The authors declare that there are no conflicts of interest regarding the publication of this paper.

\section{Acknowledgments}

The authors acknowledge the support for the work reported in this paper from the European Union's Horizon 2020 Research and Innovation Programme under the Marie Sklodowska-Curie Grant Agreement no. 642453. Greenwood Engineering, designers of the TSD are also acknowledged for their cooperation and support.

\section{References}

[1] T. Horberry, M. Halliday, and A. G. Gale, "Bridge strike reduction: optimising the design of markings", Accident Analysis \& Prevention, vol. 34, no. 5, pp. 581-588, 2002.

[2] G. Hearn and R. B. Testa, "Modal analysis for damage detection in structures," Journal of Structural Engineering, vol. 117, no. 10, pp. 3042-3063, 1991.

[3] M. Mehrjoo, N. Khaji, H. Moharrami, and A. Bahreininejad, "Damage detection of truss bridge joints using Artificial Neural Networks," Expert Systems with Applications, vol. 35, no. 3, pp. 1122-1131, 2008.

[4] P. C. Pandey and S. V. Barai, "Multilayer perceptron in damage detection of bridge structures," Computers \& Structures, vol. 54, no. 4, pp. 597-608, 1995.

[5] S. Park, C.-B. Yun, Y. Roh, and J.-J. Lee, "PZT-based active damage detection techniques for steel bridge components," Smart Materials and Structures, vol. 15, no. 4, pp. 957-966, 2006.

[6] C. K. Soh, K. K.-H. Tseng, S. Bhalla, and A. Gupta, "Performance of smart piezoceramic patches in health monitoring of a RC bridge," Smart Materials and Structures, vol. 9, no. 4, pp. 533-542, 2000.

[7] W. Fan and P. Qiao, "Vibration-based damage identification methods: a review and comparative study," Structural Health Monitoring, vol. 10, no. 1, pp. 83-111, 2011.

[8] S. Sumitoro, Y. Matsui, M. Kono, T. Okamoto, and K. Fujii, "Long span bridge health monitoring system in Japan," in Proceedings of the 6th Annual International Symposium on NDE for Health Monitoring and Diagnostics, pp. 517-524, Newport Beach, Calif, USA, 2001.

[9] I. N. Robertson, "Prediction of vertical deflections for a longspan prestressed concrete bridge structure," Engineering Structures, vol. 27, no. 12, pp. 1820-1827, 2005.

[10] J. M. Ko and Y. Q. Ni, “Technology developments in structural health monitoring of large-scale bridges," Engineering Structures, vol. 27, no. 12, pp. 1715-1725, 2005.

[11] Y.-B. Yang, C. W. Lin, and J. D. Yau, "Extracting bridge frequencies from the dynamic response of a passing vehicle," Journal of Sound and Vibration, vol. 272, no. 3-5, pp. 471-493, 2004.

[12] Y. B. Yang and C. W. Lin, "Vehicle-bridge interaction dynamics and potential applications," Journal of Sound and Vibration, vol. 284, no. 1-2, pp. 205-226, 2005.

[13] A. Malekjafarian, P. J. McGetrick, and E. J. OBrien, "A review of indirect bridge monitoring using passing vehicles," Shock and Vibration, vol. 2015, Article ID 286139, 16 pages, 2015.

[14] E. Sevillano, E. J. OBrien, and D. Martinez, "Instantaneous curvature in bridge damage detection," in Proceedings of the CSHM-6: Structural Health Monitoring of New and Ageing Infrastructure, 8 pages, Queens University, Belfast, UK, May 2016. 
[15] F. Magalhães, Á. Cunha, and E. Caetano, "Dynamic monitoring of a long span arch bridge," Engineering Structures, vol. 30, no. 11, pp. 3034-3044, 2008.

[16] J.-T. Kim and N. Stubbs, "Crack detection in beam-type structures using frequency data," Journal of Sound and Vibration, vol. 259, no. 1, pp. 145-160, 2003.

[17] E. J. OBrien and A. Malekjafarian, "A mode shape-based damage detection approach using laser measurement from a vehicle crossing a simply supported bridge," Structural Control and Health Monitoring, vol. 23, no. 10, pp. 1273-1286, 2016.

[18] Y. B. Yang, Y. C. Li, and K. C. Chang, "Constructing the mode shapes of a bridge from a passing vehicle: a theoretical study," Smart Structures and Systems, vol. 13, no. 5, pp. 797-819, 2014.

[19] Y. B. Yang and W.-F. Chen, "Extraction of bridge frequencies from a moving test vehicle by stochastic subspace identification," Journal of Bridge Engineering, vol. 21, no. 3, 2016.

[20] T. Nagayama, A. Reksowardojo, D. Su, and T. M. C. Zhang, "Bridge natural frequency estimation by extracting the common vibration component from the responses of two vehicles," in Proceedings of the 6th International Conference on Advances in Experimental Structural Engineering, Urbana-Champaign, Ill, USA, 2015.

[21] A. González, E. J. OBrien, and P. J. McGetrick, "Identification of damping in a bridge using a moving instrumented vehicle," Journal of Sound and Vibration, vol. 331, no. 18, pp. 4115-4131, 2012.

[22] A. Malekjafarian and E. J. OBrien, "On the use of a passing vehicle for the estimation of bridge mode shapes," Journal of Sound and Vibration, vol. 397, pp. 77-91, 2017.

[23] X. Kong, C. S. Cai, L. Deng, and W. Zhang, "Using dynamic responses of moving vehicles to extract bridge modal properties of a field bridge," Journal of Bridge Engineering, vol. 22, no. 6, Article ID 04017018, 9 pages, 2017.

[24] E. J. OBrien, A. Malekjafarian, and A. González, "Application of empirical mode decomposition to drive-by bridge damage detection," European Journal of Mechanics-A/Solids, vol. 61, pp. 151-163, 2017.

[25] D. Hester and A. González, "A discussion on the merits and limitations of using drive-by monitoring to detect localised damage in a bridge," Mechanical Systems and Signal Processing, vol. 90, pp. 234-253, 2017.

[26] P. J. McGetrick, D. Hester, and S. E. Taylor, "Implementation of a drive-by monitoring system for transport infrastructure utilising smartphone technology and GNSS," Journal of Civil Structural Health Monitoring, vol. 7, no. 2, pp. 175-189, 2017.

[27] C. Kim, K. Chang, S. Inoue, S. Hasegawa, and P. McGetrick, "Investigating feasibility of drive-by bridge monitoring by laboratory experiments," in Proceedings of the 24th Australian Conference on the Mechanics of Structures and Materials (ACMSM '16), pp. 1477-1484, Perth, Australia, 2016.

[28] A. Elhattab, N. Uddin, and E. OBrien, "Drive-by bridge damage monitoring using Bridge Displacement Profile Difference," Journal of Civil Structural Health Monitoring, vol. 6, no. 5, pp. 839-850, 2016.

[29] E. J. OBrien, D. Martinez, A. Malekjafarian, and E. Sevillano, "Damage detection using curvatures obtained from vehicle measurements," Journal of Civil Structural Health Monitoring, vol. 7, no. 3, pp. 333-341, 2017.

[30] E. J. OBrien, E. Sevillano, and D. Martinez, "Monitoring the condition of a bridge using a traffic speed deflectometer vehicle travelling at highway speed," in Proceedings of the in 3rd International Balkans Conference on Challenges of Civil Engineering, pp. 107-115, Tirana, Albania, 2016.

[31] G. W. Flintsch, B. Ferne, B. Diefenderfer, S. Katicha, J. Bryce, and S. Nell, "Evaluation of traffic speed continuous deflection devices," in Proceedings of the 91st Annual Meeting of Transportation Research Board, Transport Research Board, Washington, DC, USA, 2012.

[32] S. Rasmussen, J. A. Krarup, and G. Hildebrand, "Non-contact deflection measurement at high speed," in Proceedings of the 6th International Conference on the Bearing Capacity of Roads, Railways and Airfields, 8 pages, Lisbon, Portugal, 2002.

[33] M. Majka and M. Hartnett, "Effects of speed, load and damping on the dynamic response of railway bridges and vehicles," Computers \& Structures, vol. 86, no. 6, pp. 556-572, 2008.

[34] R. Karoumi, Response of Cable-Stayed and Suspension Bridges to Moving Vehicles: Analysis Methods and Practical Modeling Techniques, Department of Structural Engineering, KTH Royal Institute of Technology, Stockholm, Sweden, 1998.

[35] F. Yang and G. A. Fonder, "An iterative solution method for dynamic response of bridge-vehicles systems," Earthquake Engineering and Structural Dynamics, vol. 25, no. 2, pp. 195-215, 1996.

[36] R. W. Clough and J. Penzien, Dynamics of Structures, McGrawHill, Inc, New York, NY, USA, 1993.

[37] J. W. Tedesco, W. G. McDougal, and C. A. Ross, Structural Dynamics: Theory and Applications, Addison-Wesley, Montlo Park, Calif, USA, 1999.

[38] J. K. Sinha, M. I. Friswell, and S. Edwards, "Simplified models for the location of cracks in beam structures using measured vibration data," Journal of Sound and Vibration, vol. 251, no. 1, pp. 13-38, 2002.

[39] F. N. Catbas, M. Gul, and J. L. Burkett, "Conceptual damagesensitive features for structural health monitoring: laboratory and field demonstrations," Mechanical Systems and Signal Processing, vol. 22, no. 7, pp. 1650-1669, 2008.

[40] W.-Y. He and S. Zhu, "Moving load-induced response of damaged beam and its application in damage localization," Journal of Vibration and Control, vol. 22, no. 16, pp. 3601-3617, 2016.

[41] F. Tyan, Y. F. Hong, and S. H. Tu, "Generation of random road profiles," International Journal of Advanced Engineering Technology, vol. 4, no. 2, pp. 1373-1378, 2009.

[42] A. González, E. Covián, M. Casero, and M. Celemín, "Evaluation of the true behavior of the end supports in the Carbajal de la Legua old bridge," in Proceedings of the Istambul Bridge Conference, pp. 187-197, Istambul, Turkey, 2014.

[43] M. Johansmann, G. Siegmund, and M. Pineda, "Targeting the limits of laser Doppler vibrometry," in Proceedings of the IDEMA, pp. 1-12, 2005.

[44] G. R. Rada, S. Nazarian, B. A. Visintine, R. V. Siddharthan, and N. Sivaneswaran, "Use of high-speed deflection devices in network-level PMS applications: are we ready?" in Proceedings of the 9th International Conference on Managing Pavement Assets, EEUU, Alexandria, Va, USA, 2015.

[45] P. Podder, T. Z. Khan, M. H. Khan, and M. M. Rahman, "Comparative performance analysis of hamming, hanning and blackman window," International Journal of Computer Applications, vol. 96, no. 18, pp. 1-6, 2014. 


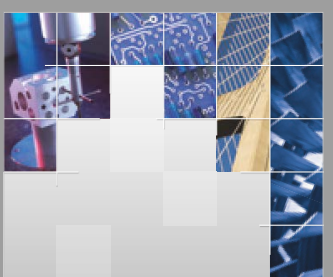

\section{Enfincering}
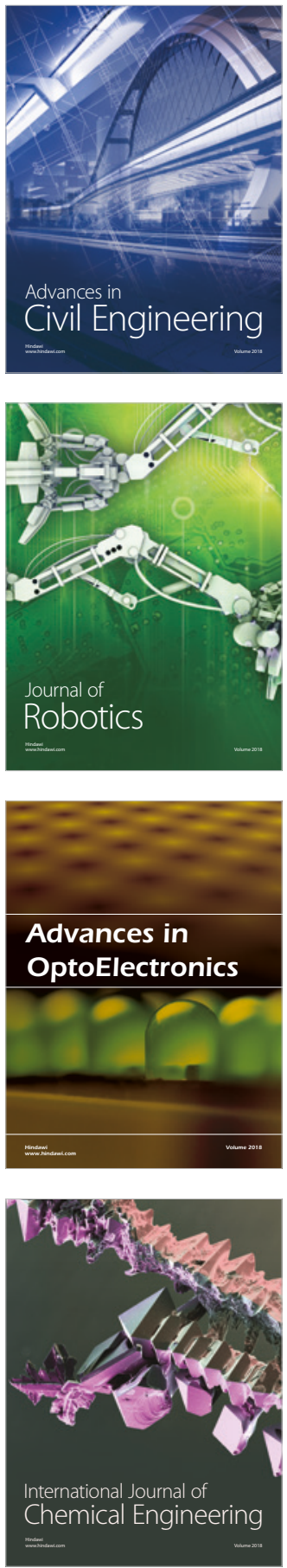

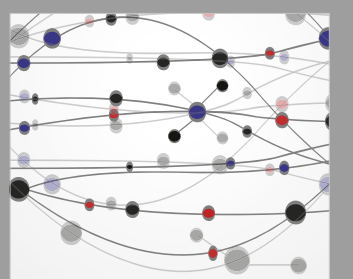

\section{Rotating \\ Machinery}

The Scientific World Journal

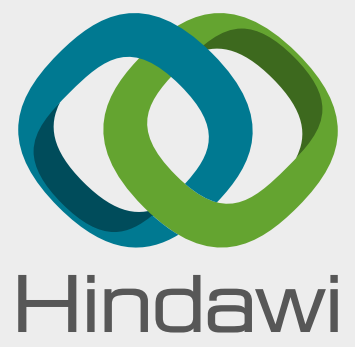

Submit your manuscripts at

www.hindawi.com
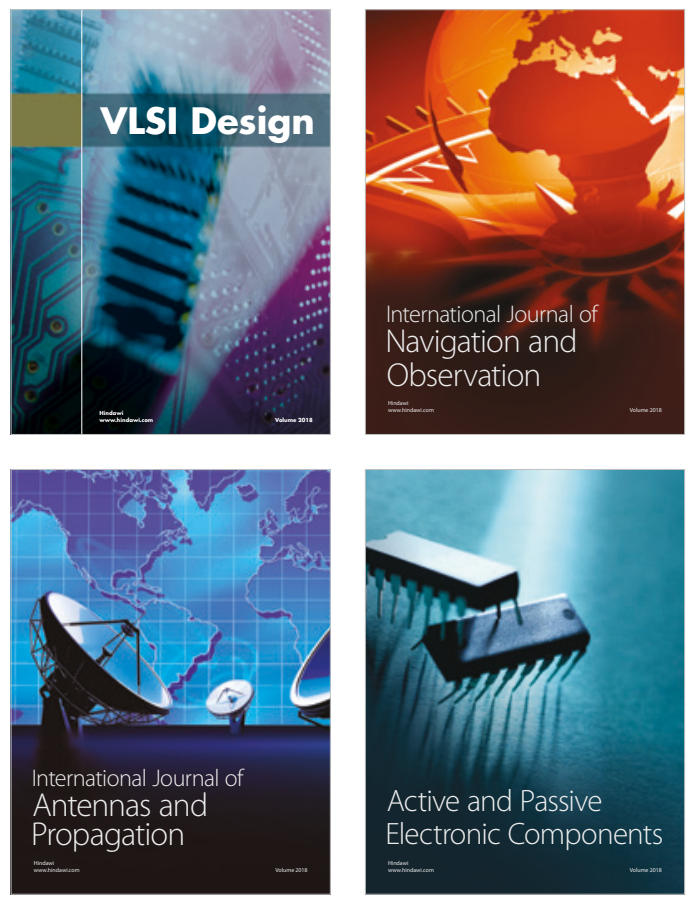
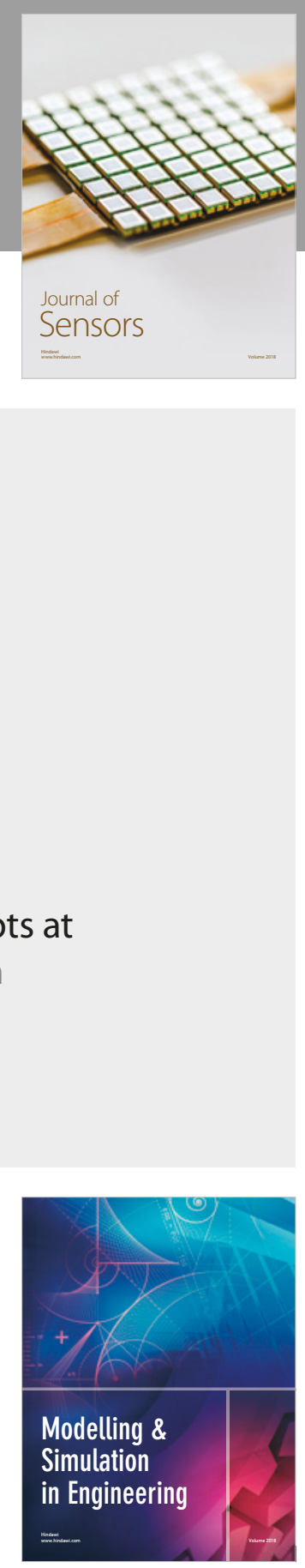

\section{Advances \\ Multimedia}
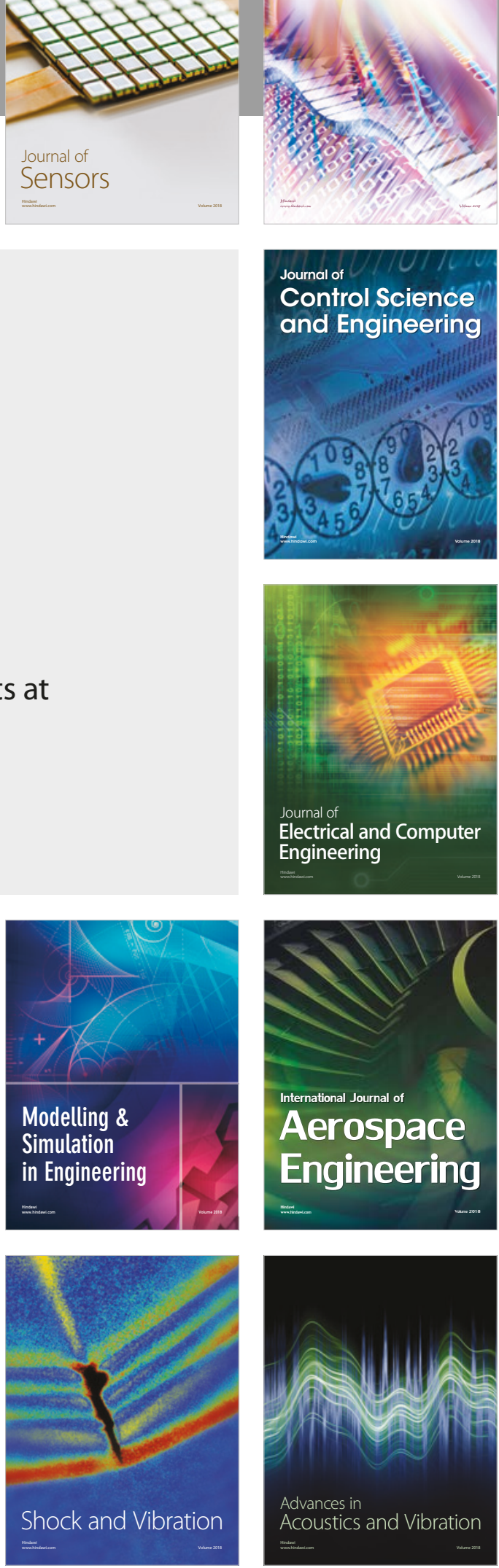